\title{
Estimativa de Parâmetros Genéticos para Características de Carcaça em um Rebanho de Suínos Large White ${ }^{1}$
}

\section{Gastón Andrés Fernandez Giné2 ${ }^{2}$, Rilke Tadeu Fonseca de Freitas ${ }^{3}$, Antonio Ilson Gomes de Oliveira $^{4}$, Idalmo Garcia Pereira ${ }^{5}$, Tarcísio de Moraes Gonçalves ${ }^{6}$}

\begin{abstract}
RESUMO - Com o objetivo de estimar parâmetros genéticos para características de carcaça de suínos, foram utilizados dados de rendimento de carcaça (RC), comprimentos de carcaça pelo método Brasileiro (CCMB) e pelo método americano (CCMA), espessuras de toucinho média $(\mathrm{ETM})$ e a $6,5 \mathrm{~cm}$ da linha dorsal $\left(\mathrm{P}_{2}\right)$, área de olho de lombo (AOL), relação carne:gordura (RCG), rendimento de pernil (RP), porcentagens de carne (PC), gordura (PG) e cortes magros (PCM), relação peso da gordura/peso da carne (RGC) e taxa de crescimento em músculo (TCM), obtidos de 711 suínos Large White. Os componentes de (co)variância foram estimados pelo método da Máxima Verossimilhança Restrita (REML), incluindo no modelo efeitos fixos (sexo e grupo contemporâneo) e aleatórios (efeitos genéticos e residuais). Utilizou-se a covariável "peso de abate" para RC e "peso da carcaça fria" para CCMB, CCMA, ETM, $\mathrm{P}_{2}, \mathrm{AOL}$ e RCG. Estimativas de $\mathrm{h}^{2}$ para RC, CCMB, CCMA, ETM, $\mathrm{P}_{2}$, AOL, RCG, RP, PC, PG, PCM, RGC e TCM foram, respectivamente, $0,39,0,34,0,19,0,17,0,16,0,27,0,15,0,12,0,45,0,36,0,32,0,39$ e 0,37 . Espessura de toucinho média, $\mathrm{P}_{2}$ e RCG foram as características mais correlacionadas geneticamente com carne e gordura da carcaça, seguidas da AOL, CCMA e RP, com tendência similar para as correlações fenotípicas. AOL e RCG tiveram maiores correlações genéticas e fenotípicas com TCM, ou seja, melhoram, por intermédio de seleção, a qualidade da carcaça do animal e a taxa de produção de carne.
\end{abstract}

Palavras-chave: parâmetros genéticos, REML, suínos, carcaça

\section{Estimate of Genetic Parameters for Carcass Traits in Large White Swine Herd}

ABSTRACT - In order to estimate genetic parameters for swine carcasses traits, data of dressing percentage (DP), carcass length by Brazilian method (CLBM) and American method (CLAM), average backfat thickness (ABT), backfat thickness at $6.5 \mathrm{~cm}$ from the dorsal line $\left(\mathrm{P}_{2}\right)$, loin eye area (LEA), lean:fat ratio (LFR), ham yield (HY), lean, fat and lean cuts yields (LY, FY and LCY), fat weight:lean weight ratio (FLR), and lean tissue growth rate (LTGR) obtained from 711 swine of the Large White breed, were used. (Co)variances components were estimated by the Restricted Maximum Likelyhood Method (REML) including in the model fixed effects of sex and contemporary group, and random effects of animal. Covariables "slaughter weight" for DP and "cold carcass weight" for CLBM, CLAM, ABT, $\mathrm{P}_{2}$, LEA and LFR, were used. For DP, CLBM, CLAM, ABT, $\mathrm{P}_{2}$, LEA, LFR, HY, LY, FY, LCY, FLR and LTGR, the estimates of $\mathrm{h}^{2}$ were $0.39,0.34,0.19,0.17,0.16,0.27,0.15,0.12,0.45,0.36,0.32,0.39$ and 0.37 , respectively. Backfat thickness, $\mathrm{P}_{2}$ and LFR were the most genetically correlated traits with the carcass fat and meat contents and LEA, CLAM and HY showed intermediary values. In general, the same trend was observed for the phenotypic correlations. LEA and LFR showed greatest genetic and phenotypic correlation with LTGR, concluding that there is improve of animal carcass quality and the lean production rate through selection.

Key Words: genetic parameter, REML, swine, carcass

\section{Introdução}

O sucesso de um programa de melhoramento genético depende, em sua elaboração, do conhecimento de estimativas precisas e confiáveis dos parâmetros genéticos, fenotípicos e ambientais da população a ser melhorada. O método da máxima verossimilhança restrita (REML), proposto por Patterson \& Thompson (1971), é o mais recomendado para estimação de componentes de (co)variância em animais, pois, além de considerar a perda de graus de liberdade resultante dos efeitos fixos, as estimativas são mantidas sempre dentro do espaço paramétrico (Anderson, 1984; Costa et al., 2001).

\footnotetext{
${ }^{1}$ Parte da dissertação de mestrado do primeiro autor apresentada ao Programa de Pós-Graduação em Zootecnia da UFLA. Projeto financiado parcialmente pela FAPEMIG e pelo CNPq.

2 Zootecnista, M.Sc. pelo DZO da UFLA. E.mail: gastongine @ yahoo.com.br

3 Professor Adjunto do DZO da UFLA - CP 37 - 37200-000 - Lavras - MG. E.mail: rilke@ufla.br

4 Professor Titular Aposentado da UFLA. E.mail: ailson@ufla.br

5 Professor Adjunto da FAFEOD. E.mail: idalmo@fafeod.br

6 Professor Adjunto do DZO da UFLA. E.mail: tarcisio@ufla.br
} 
Dentre as diversas características de importância econômica na exploração de suínos, as de carcaça e desempenho em deposição de carne têm, nos últimos anos, merecido grande atenção. Isso se deve ao pagamento baseado na tipificação e bonificação das carcaças, realizado pela indústria de processamento de carne suína, que passou a exigir carcaças com maior quantidade de carne (em porcentagem e peso), menor quantidade de gordura e qualidade adequada para o processamento industrial. Além disso, animais mais eficientes na deposição de tecido protéico (maior velocidade de crescimento muscular) podem reduzir os custos de produção, tornando a carne suína mais acessível ao consumidor e mais rentável ao produtor.

As características de classificação de carcaça, principalmente aquelas de fácil mensuração, são ferramentas importantes para serem usadas como critério de seleção. Desta forma, é essencial conhecer as estimativas de herdabilidade destas características e as correlações existentes entre estas e os teores de carne e gordura da carcaça, relacionadas à qualidade da carcaça e à taxa de crescimento em músculo, associada ao desempenho.

Assim, objetivou-se com o presente trabalho estimar parâmetros genéticos de características de classificação e dissecação de carcaças e da taxa de crescimento em músculo, em um rebanho de suínos da raça Large White.

\section{Material e Métodos}

Os dados para este trabalho foram coletados durante o período de maio de 1997 a março de 2000, em suínos da raça Large White provenientes do setor de Suinocultura do Departamento de Zootecnia da Universidade Federal de Lavras (UFLA), situada no município de Lavras, na região sul do Estado de Minas Gerais.

Foram avaliados 711 suínos ( 371 machos castrados e 340 fêmeas) da raça Large White, oriundos do acasalamento ao acaso de 12 cachaços e 46 porcas do rebanho da UFLA. Os animais foram abatidos ao atingir, em média, $95 \mathrm{~kg}$ de peso vivo, e suas meiacarcaças esquerdas, após resfriamento em câmara fria à temperatura de 2 a $4^{\circ} \mathrm{C}$ por 24 horas, foram pesadas e classificadas segundo o Método Brasileiro de Classificação de Carcaças descrito pela ABCS (1973). Além disso, foram tomadas as medidas de comprimento de carcaça pelo método americano (CCMA), conforme Boggs \& Merkel (1979), e espessura de toucinho a $6,5 \mathrm{~cm}$ da linha dorsal na altura da última costela $\left(\mathrm{P}_{2}\right)$, de acordo com Cuthbertson \& Pease (1968). A seguir, as carcaças foram dissecadas de acordo com a técnica de Cuthbertson (1968), modificada por Almeida Neto (1992).

Foram analisadas as seguintes características de classificação de carcaça: rendimento de carcaça (RC); comprimento de carcaça pelo MBCC (CCMB); comprimento de carcaça pelo método americano (CCMA); espessura de toucinho média (ETM); espessura de toucinho a $6,5 \mathrm{~cm}$ da linha dorsal $\left(\mathrm{P}_{2}\right)$; área de olho de lombo (AOL); relação carne:gordura (RCG) e rendimento de pernil (RP). Foram obtidas, por intermérdio da dissecação, as porcentagens de carne (PC), gordura (PG) e cortes magros (PCM) e relação peso da gordura/peso da carne (RGC).

Além dessas características de carcaça, foram avaliados o ganho de peso médio diário (GPMD) e a taxa de crescimento em músculo (TCM), sendo a primeira calculada como GPMD $=($ Peso vivo ao abate - Peso vivo no início da fase de crescimento)/ Pe e a segunda calculada pela fórmula desenvolvida por Fowler et al. (1976): TCM = (MF - MI) / Pe, em que MF é o peso do músculo ao abate; MI, o peso do músculo no início da fase de crescimento; e Pe, o período transcorrido, em dias, do início da fase de crescimento até o abate.

As estimativas de MF e MI são dadas por:

$\mathrm{MF}=$ Peso de abate $\times \mathrm{RC} \times \mathrm{RF} \times \mathrm{PC} \times 10^{-6}$, sendo RF o Rendimento de Frigorificação;

MI $=-1,59+(0,44 \times$ Peso ao início da fase de crescimento), segundo Brannaman et al. (1984).

Foram mantidos apenas os animais que possuíam informações de todas as características, o que reduziu o número total de registros para 684 (356 machos e 328 fêmeas).

Para as análises envolvendo TCM e GPMD, foram mantidos apenas 512 animais (254 machos e 258 fêmeas), progênies de 41 matrizes e 11 reprodutores.

Os componentes de (co)variância foram estimados pelo método da Máxima Verossimilhança Restrita (REML), utilizando-se o programa computacional MTDFREML (Boldman, et al., 1995) e adotando-se como critério de precisão, a variância dos valores assumidos pela verossimilhança nos pontos do Simplex inferiores a $10^{-9}$.

Foram efetuadas análises de característica única (univariadas) para todas as características avaliadas, e análise conjunta de duas características (bivariadas) 
apenas para os pares formados pela combinação das características de classificação de carcaça com as de dissecação, ganho de peso e taxa de crescimento em músculo.

Os grupos contemporâneos foram formados por intermédio da combinação de estação ( 1 = janeiro a março; $2=$ abril a junho; $3=$ julho a setembro; 4 = outubro a dezembro) e ano de nascimento (1997 a 2000).

Consideraram-se como efeitos fixos, o sexo e o grupo contemporâneo, e como efeitos aleatórios, o efeito genético direto do animal e o efeito residual. Como covariável, utilizou-se o peso de abate para rendimento de carcaça $(\mathrm{RC})$, e o peso da meia carcaça esquerda fria para as demais características que, em análises preliminares, foram significativamente influenciadas por esta variável (CCMB, CCMA, ETM, $\mathrm{P}_{2}$, AOL, RCG).

O modelo utilizado na análise, na sua forma matricial, foi o seguinte:

$$
\underset{\sim}{y}=\underset{\sim}{\beta} \underset{\sim}{\beta} \underset{\sim}{\mathrm{a}}+\underset{\sim}{e}
$$

em que y é vetor das variáveis dependentes; $\mathrm{X}$, a matriz de incidência dos efeitos fixos e covariáveis; b, o vetor dos efeitos fixos e covariáveis; Z, a matriz de incidência dos valores genéticos; a, o vetor dos valores genéticos dos indivíduos; e, o vetor dos erros pressupostos NID $\left(0, \sigma_{\mathrm{e}}^{2}\right)$. Assumiu-se que $\mathrm{E}(\mathbf{a})=\mathbf{0}$ e $\operatorname{Var}(\mathbf{a})=\mathbf{A} \sigma^{2}{ }_{a}$, em que $\mathbf{A}$ é a matriz de parentesco e $\sigma^{2}$ a o componente de variância genética aditiva.

O efeito comum de leitegada não foi considerado no modelo, devido ao pequeno tamanho do arquivo de dados, tendo sido detectado confundimento entre os efeitos fixos e os efeitos aleatórios na presença deste, obtendo-se estimativas com valores extremamente fora daqueles encontrados quando este não foi considerado, e dos relatados na literatura.

\section{Resultados e Discussão}

As médias, com os respectivos desvios-padrão, das características de carcaça dos suínos avaliados no experimento (Tabela 1) evidenciam que os animais avaliados apresentaram elevada espessura de toucinho, não podendo ser comparados com animais selecionados, atualmente produzidos, uma vez que este rebanho não foi submetido a qualquer programa de seleção. A média encontrada para ETM e $\mathrm{P}_{2}$ foi maior que as obtidas por Catalan (1986) e Larzul et al. (1997). Pesquisas com outras raças indicam ETM variando de 21 a $40 \mathrm{~mm}$ (Rahnefeld et al., 1983 e Oliveira, 1988), evidenciando que estes animais possuem carcaças de baixa qualidade quanto à espessura de toucinho.

Tabela 1 - Número de observações (n), médias e desviospadrão das características de carcaças de suínos Large White

Table 1 - Number of records ( $n$ ), means and standard deviations of traits of the Large White swine carcass

\begin{tabular}{|c|c|c|c|}
\hline $\begin{array}{l}\text { Características }{ }^{1} \\
\text { Traits }\end{array}$ & $\mathrm{N}$ & $\begin{array}{l}\text { Média } \\
\text { Mean }\end{array}$ & $\begin{array}{r}\text { Desvio-padrão } \\
\text { Standard deviation }\end{array}$ \\
\hline $\begin{array}{l}\mathrm{PV}(\mathrm{kg}) \\
L W\end{array}$ & 684 & 94,84 & 3,97 \\
\hline $\begin{array}{l}\mathrm{RC}(\%) \\
\mathrm{DP}\end{array}$ & 684 & 81,30 & 2,76 \\
\hline $\begin{array}{l}\text { CCMB }(\mathrm{cm}) \\
C L B M\end{array}$ & 684 & 93,23 & 3,15 \\
\hline $\begin{array}{l}\text { CCMA }(\mathrm{cm}) \\
\text { CLAM }\end{array}$ & 684 & 78,23 & 2,87 \\
\hline $\begin{array}{l}\mathrm{ETM}(\mathrm{mm}) \\
A B T\end{array}$ & 684 & 35,35 & 5,02 \\
\hline $\begin{array}{l}\mathrm{P}_{2}(\mathrm{~mm}) \\
\mathrm{AOL}\left(\mathrm{cm}^{2}\right)\end{array}$ & $\begin{array}{l}684 \\
684\end{array}$ & $\begin{array}{l}24,87 \\
31,96\end{array}$ & $\begin{array}{l}6,96 \\
4,59\end{array}$ \\
\hline $\begin{array}{l}L E A \\
\text { RCG }\end{array}$ & 684 & 0,77 & 0,18 \\
\hline $\begin{array}{l}M F R \\
\operatorname{RP}(\%) \\
H Y\end{array}$ & 684 & 30,61 & 1,79 \\
\hline $\begin{array}{l}\mathrm{PC}(\%) \\
L M Y\end{array}$ & 684 & 50,30 & 3,20 \\
\hline $\begin{array}{l}\mathrm{PG}(\%) \\
F Y\end{array}$ & 684 & 27,63 & 3,46 \\
\hline $\begin{array}{l}\operatorname{PCM}(\%) \\
L C Y\end{array}$ & 684 & 50,26 & 3,14 \\
\hline $\begin{array}{l}\text { RGC } \\
F M R\end{array}$ & 684 & 0,55 & 0,10 \\
\hline $\begin{array}{l}\text { GPMD } \\
D L W G\end{array}$ & 512 & 0,79 & 0,14 \\
\hline $\begin{array}{l}\text { TCM }(\mathrm{kg} / \text { dia }) \\
L T G R(k g / \text { day })\end{array}$ & 512 & 0,32 & 0,06 \\
\hline
\end{tabular}

${ }^{1} \mathrm{PV}$ : Peso vivo; RC: Rendimento de carcaça; CCMB: Comprimento de carcaça pelo MBCC; CCMA: Comprimento de carcaça pelo método americano; ETM: Espessura de toucinho média; $P_{2}$ : Espessura de toucinho à $6,5 \mathrm{~cm}$ da linha do dorso; AOL: Área de olho de lombo; RCG: Relação carne:gordura; RP: Rendimento de pernil; PC: Porcentagem de carne; PG: Porcentagem de gordura; PCM: Porcentagem de cortes magros; RGC: Relação gordura:carne; GPMD: Ganho de peso médio diário; TCM: Taxa de crescimento em músculo

${ }^{1}$ LW: Live weight; DP: dressing percentage; CLBM: carcass length by Brazilian method; CLAM: carcass length by American method; ABT: average backfat thickness; $P_{2}$ : backfat thickness at $6.5 \mathrm{~cm}$ from the dorsal line: LEA: Loin eye area; MFR: lean:fat ratio; HY: ham yield; LMY: lean meat yield; FY: fat yield; $L C Y$ : lean cuts yield; FLR: fat:lean ratio; DLWG: daily live weight gain; LTGR: lean tissue growth rate 
As demais características de classificação de carcaça apresentaram valores médios que estão dentro dos limites descritos na literatura. Com relação às características de dissecação avaliadas neste estudo, verificaram-se médias coerentes com as pesquisas realizadas por Larzul et al. (1997). O ganho médio diário de peso e a taxa de crescimento em músculo, também apresentaram valores semelhantes aos observados na literatura, sendo que para estes últimos, variaram de 0,26 a 0,37 kg/dia (Bereskin, 1987; Stern et al., 1993; Larzul et al., 1997).

As estimativas de herdabilidade para as características de carcaça, obtidas nas análises univariadas e bivariadas, são apresentadas na Tabela 2. Considerando que as estimativas de herdabilidade foram semelhantes entre as análises, os resultados serão discutidos independentemente do tipo de análise realizada.
Dentre as características de classificação de carcaça, RC, CCMB e AOL apresentaram as maiores estimativas de herdabilidade, sugerindo maior progresso genético nestas características em processos seletivos. Os valores encontrados para RC assemelham-se aos obtidos por Hicks et al. (1998), enquanto para o CCMB as estimativas de herdabilidade foram próximas às encontradas por Collao Saenz (1994) e menores que as obtidas por Roso et al. (1995) e Hicks et al. (1998).

O valor de herdabilidade estimado para a AOL foi semelhante aos apresentados por Johnson et al. (1999) e inferior aos estimados por Larzul et al. (1997) e Hicks et al. (1998).

Para as demais características de classificação, as estimativas de herdabilidade variaram de 0,12 a 0,19 , demonstrando que estas características apre-

\begin{tabular}{|c|c|c|c|}
\hline $\begin{array}{l}\text { Heritc } \\
\text { analy }\end{array}$ & $s\left(h^{2}\right)$ for swine cal & traits in & and bivariate \\
\hline \multirow{2}{*}{$\begin{array}{l}\text { Características }{ }^{1} \\
\text { Traits }\end{array}$} & \multirow{2}{*}{$\begin{array}{c}\text { Análise univariada } \\
\text { Univariate analysis }\end{array}$} & \multicolumn{2}{|c|}{$\begin{array}{l}\text { Análise bivariada } \\
\text { Bivariate analysis }\end{array}$} \\
\hline & & $\begin{array}{c}\text { Média } \\
\text { Mean }\end{array}$ & $\begin{array}{c}\text { Intervalo } \\
\text { Interval }\end{array}$ \\
\hline $\mathrm{RC}$ & 0,39 & 0,38 & $0,37-039$ \\
\hline CCMB & 0,34 & 0,34 & $0,33-0,34$ \\
\hline & 0,19 & 0,18 & $0,18-0,19$ \\
\hline $\begin{array}{l}\text { CLAM } \\
\text { ETM }\end{array}$ & 0,17 & 0,16 & $0,15-0,16$ \\
\hline $\mathrm{P}_{2}$ & $\begin{array}{l}0,16 \\
0,27\end{array}$ & $\begin{array}{l}0,16 \\
0,27\end{array}$ & $\begin{array}{l}0,15-0,16 \\
0,27-0,27\end{array}$ \\
\hline LEA & & & \\
\hline RCG & 0,15 & 0,14 & $0,14-0,15$ \\
\hline $\begin{array}{l}\mathrm{RP} \\
H Y\end{array}$ & 0,12 & 0,11 & $0,10-0,11$ \\
\hline $\mathrm{PC}$ & 0,45 & 0,45 & $0,42-0,46$ \\
\hline $\begin{array}{l}\mathrm{PG} \\
F P\end{array}$ & 0,36 & 0,36 & $0,34-0,37$ \\
\hline PCM & 0,32 & 0,32 & $0,31-0,32$ \\
\hline $\begin{array}{l}\mathrm{RGC} \\
F M R\end{array}$ & 0,39 & 0,39 & $0,37-0,40$ \\
\hline GPMD & 0,41 & 0,41 & $0,39-0,42$ \\
\hline $\begin{array}{l}\text { TCM } \\
L T G R\end{array}$ & 0,28 & 0,28 & $0,27-0,30$ \\
\hline
\end{tabular}

${ }^{1}$ Definidos na Tabela 1 (According to Table 1). 
sentaram baixa variabilidade genética no rebanho estudado. É importante ressaltar o pequeno número de reprodutores utilizados na formação deste rebanho, que pode elevar o coeficiente de endogamia, comprometendo a inferência destes resultados para outros rebanhos.

Em média, as características de dissecação apresentaram estimativas de herdabilidade superiores àquelas estimadas para as características de classificação e, entre todas as características de carcaça estudadas, a PC foi a de maior herdabilidade. Os valores de herdabilidade obtidos para PC, PCM, PG e RGC variaram de 0,32 a 0,45 . Estes resultados não só estão coerentes com os obtidos anteriormente (Hofer et al., 1992; Collao Saenz, 1994; Larzul et al., 1997; Sonesson et al., 1998), como também são alicerçados pela natureza genética dessas características (genes de efeitos predominantemente aditivos).

As estimativas de herdabilidade obtidas para GPMD e TCM foram elevadas, sendo semelhantes às encontradas por Stern et al. (1993), Chen et al. (1994) e Cameron (1994), evidenciando a possibilidade de haver grande progresso genético neste rebanho, em programas de melhoramento com o propósito de se aumentar o ganho de peso e a taxa de deposição de carne na carcaça.

Apesar das características de dissecação, GPMD e TCM possuírem, em média, maior herdabilidade que as de classificação, vale ressaltar que, a obtenção dos dados de dissecação, necessários também para o cálculo da TCM, exige muita mão-de-obra e tempo e ocasiona cortes indevidos na carcaça, comprometendo a sua comercialização. Dessa forma, este processo é oneroso e praticamente inviável para implantação em grande escala. Por outro lado, as características de classificação são mais facilmente mensuradas, principalmente aquelas que podem ser medidas por ultra-som, portanto, sem a necessidade de abater os animais.

As estimativas de correlações genéticas e fenotípicas das características de classificação com as de dissecação e taxa de crescimento em músculo são apresentadas nas Tabelas 3 e 4, respectivamente.

As características de classificação que tiveram maior correlação genética com as de dissecação, foram as espessuras de toucinho $\left(\mathrm{ETM}\right.$ e $\left.\mathrm{P}_{2}\right)$ e a relação carne:gordura (RCG), sendo a espessura de toucinho média (ETM) de maior magnitude e altamente correlacionada com o conteúdo de gordura da carcaça. Verifica-se que as características ETM, $\mathrm{P}_{2}$ e RCG foram negativamente correlacionadas com as medidas que avaliam a quantidade de carne na carcaça (PC e PCM) e positivamente correlacionadas com as medidas que avaliam a quantidade de gordura $(\mathrm{PG}$

Tabela 3 - Correlações genéticas $\left(r_{G}\right)$ entre características de classificação e dissecação de carcaça, e taxa de crescimento em músculo de suínos da raça Large White

Table 3 - Genetic correlation $\left(r_{G}\right)$ among traits of carcass classification and dissection and lean tissue growth rate in Large White pigs

\begin{tabular}{|c|c|c|c|c|c|c|}
\hline $\begin{array}{l}\text { Características }{ }^{1} \\
\text { Traits }\end{array}$ & $\begin{array}{l}\mathrm{PC} \\
L P\end{array}$ & $\begin{array}{l}\mathrm{PG} \\
F P\end{array}$ & $\begin{array}{l}\text { PCM } \\
L C P\end{array}$ & $\begin{array}{l}\text { RGC } \\
F M R\end{array}$ & $\begin{array}{l}\text { TCM } \\
L T G R\end{array}$ & $\begin{array}{l}\text { GPMD } \\
D L W G\end{array}$ \\
\hline $\begin{array}{l}\mathrm{RC} \\
\mathrm{DP}\end{array}$ & 0,12 & $-0,02$ & 0,03 & $-0,06$ & 0,26 & 0,00 \\
\hline $\begin{array}{l}\text { CCMB } \\
C L B M\end{array}$ & 0,02 & $-0,10$ & 0,22 & $-0,07$ & $-0,30$ & $-0,10$ \\
\hline $\begin{array}{l}\text { CCMA } \\
C L A M\end{array}$ & 0,13 & $-0,31$ & 0,48 & $-0,26$ & $-0,29$ & $-0,08$ \\
\hline $\begin{array}{l}\text { ETM } \\
A B T\end{array}$ & $-0,86$ & 0,92 & $-0,78$ & 0,92 & 0,24 & 0,75 \\
\hline $\begin{array}{l}\mathrm{P}_{2} \\
\mathrm{AOL} \\
L E A\end{array}$ & $\begin{array}{c}-0,69 \\
0,41\end{array}$ & $\begin{array}{c}0,66 \\
-0,29\end{array}$ & $\begin{array}{l}-0,77 \\
0,42\end{array}$ & $\begin{array}{l}0,68 \\
-0,31\end{array}$ & $\begin{array}{l}0,17 \\
0,40\end{array}$ & $\begin{array}{r}0,48 \\
-0,24\end{array}$ \\
\hline $\begin{array}{l}\mathrm{RCG} \\
M F R\end{array}$ & $-0,67$ & 0,70 & $-0,56$ & 0,72 & $-0,53$ & 0,21 \\
\hline $\begin{array}{l}\mathrm{RP} \\
\mathrm{HY}\end{array}$ & $-0,23$ & 0,30 & $-0,28$ & 0,30 & 0,06 & 0,32 \\
\hline
\end{tabular}

R. Bras. Zootec., v.33, n.2, p.337-343, 2004 
Tabela 4 - Correlações fenotípicas $\left(r_{P}\right)$ entre características de classificação e dissecação de carcaça e taxa de crescimento em músculo de suínos da raça Large White

Table 4 - Phenotipic correlation $\left(r_{P}\right)$ among traits of carcass classification and dissection, and lean tissue growth rate in Large White pigs

\begin{tabular}{|c|c|c|c|c|c|c|}
\hline Características ${ }^{1}$ & $\mathrm{PC}$ & $\mathrm{PG}$ & PCM & $\mathrm{RGC}$ & TCM & GPMD \\
\hline Traits & $L P$ & $F P$ & $L C P$ & $F M R$ & $L T G R$ & $D L W G$ \\
\hline $\mathrm{RC}$ & $-0,05$ & 0,10 & $-0,10$ & 0,08 & 0,05 & $-0,08$ \\
\hline \multicolumn{7}{|l|}{$D P$} \\
\hline CCMB & 0,15 & $-0,21$ & 0,25 & $-0,20$ & $-0,04$ & $-0,12$ \\
\hline \multicolumn{7}{|l|}{ CLBM } \\
\hline CCMA & 0,18 & $-0,23$ & 0,30 & $-0,22$ & $-0,03$ & $-0,14$ \\
\hline \multicolumn{7}{|l|}{ CLAM } \\
\hline ETM & $-0,52$ & 0,60 & $-0,54$ & 0,58 & 0,01 & 0,14 \\
\hline \multicolumn{7}{|l|}{$A B T$} \\
\hline $\mathrm{P}_{2}$ & $-0,57$ & 0,66 & $-0,64$ & 0,64 & $-0,02$ & 0,18 \\
\hline $\mathrm{AOL}$ & 0,48 & $-0,46$ & 0,47 & $-0,47$ & 0,21 & $-0,07$ \\
\hline \multicolumn{7}{|l|}{$L E A$} \\
\hline RCG & $-0,59$ & 0,63 & $-0,60$ & 0,64 & $-0,16$ & 0,00 \\
\hline \multicolumn{7}{|l|}{$M F R$} \\
\hline $\mathrm{RP}$ & 0,25 & $-0,17$ & 0,29 & $-0,21$ & 0,12 & 0,00 \\
\hline$H Y$ & & & & & & \\
\hline
\end{tabular}

1 Definidos na Tabela 1 (According to Table 1).

e RGC), concordando com os resultados encontrados por Bereskin (1987), Almeida Neto (1992), Collao Saenz (1994) e Sonessonet al. (1998), demonstrando que podem ser boas indicadoras da qualidade de carcaça.

As características AOL e RP demonstraram que são geneticamente correlacionadas com as características de dissecação, sendo que a AOL apresentou correlações positivas com PC e PCM e negativas com PG e RGC, corroborando os resultados obtidos por Bereskin (1987) e Collao Saenz (1994).

Embora o CCMB tenha apresentado correlações genéticas muito baixas com as medidas de dissecação, o CCMA apresentou correlações genéticas um pouco maiores, principalmente com PG e PCM, demonstrando que animais mais compridos poderão produzir carcaças de melhor qualidade, o que corrobora os resultados obtidos por Collao Saenz (1994).

A AOL e a RCG tiveram as maiores correlações genéticas com a TCM. Dessa forma, pode-se esperar que a seleção para aumentar a AOL ou diminuir a RCG, além de proporcionar uma carcaça de melhor qualidade, ocasionaria melhor desempenho para a produção de carne deste rebanho.

As espessuras de toucinho e comprimentos de carcaça apresentaram correlações genéticas intermediárias e desfavoráveis com TCM, ou seja, ao tentar melhorar a qualidade da carcaça destes ani- mais, reduzindo a espessura de toucinho ou aumentando o comprimento da carcaça, o desempenho da produção de carne na carcaça tenderia a piorar, e vice-versa. Para contornar este antagonismo e obter progresso para ambos objetivos, recomenda-se trabalhar com índices de seleção que ponderem adequadamente estas características.

As correlações fenotípicas entre todas as características, com exceção do rendimento de carcaça e pernil, tiveram o mesmo sentido que as correlações genéticas, divergindo apenas em magnitude.

\section{Conclusões}

É possível obter ganhos genéticos em processos seletivos que considerem as variáveis rendimento de carcaça, comprimento de carcaça pelo MBCC, área de olho de lombo e também as características de dissecação e taxa de crescimento em músculo.

Processos seletivos para espessura de toucinho média e/ou $\mathrm{P}_{2}$, embora produzam carcaças de melhor qualidade, podem reduzir a taxa de crescimento em músculo.

Os parâmetros estimados para a área de olho de lombo evidenciam a importância desta característica na obtenção de bons resultados para o melhoramento das carcaças e produção de carne deste rebanho. 


\section{Literatura Citada}

ALMEIDA NETO, P.P. Parâmetros genéticos e fenotípicos de características de carcaça de suínos. Lavras: Universidade Federal de Lavras, 1992. 71p. Dissertação (Mestrado em Zootecnia) - Universidade Federal de Lavras, 1992.

ANDERSON, R.D. Variance components. In: QUAAS, R.L.; ANDERSON, R.D.; GILMOUR, A.R. (Eds.) Use of mixed models for prediction and for estimation of (co) variance components. [s.1.] University of New England-AGBU, 1984. p.77-145. (BLUP School Handbook)

ASSOCIAÇÃ̃O BRASILEIRA DE CRIADORES DE SUÍNOS. Método Brasileiro de Classificação de Carcaça. Estrela, RS: ABCS, 1973. 17p.

BERESKIN, B. Genetic and phenotypic parameters for pig growth and body composition estimated by intraclass correlation and parent-offspring regression. Journal of Animal Science, v.64, p.1619-1629, 1987.

BOGGS, D.L.; MERKEL, R.A. Live animal carcass evaluation and selection manual. Toronto: Kendall/Hunt, 1979. 199p.

BOLDMAN, K.G.; KRIESE, S.; Van VLECK, L.D. et al. MTDFREML user's guide USDA/ARS Claycenter, Nebrasca, 1995. 114p.

BRANNAMAN, J.L.; CHRISTIAN, L.L.; ROTHSCHILD, M.F. et al. Prediction equations for estimating lean quantity in 15.0 to $50.0 \mathrm{~kg}$ pigs. Journal of Animal Science, v.59, n.4, p.991-996, 1984.

CAMERON, N.D. Selection for components of efficient lean growth rate in pigs. 1. Selection pressue applied and responses in a Large White herd. Animal Production, v.59, p.251-262, 1994.

CATALAN, G. Estimativa de parâmetros genéticos e fenotípicos em suínos Landrace, Large White e Duroc, nas fases de crescimento e terminação. Viçosa, MG: Universidade Federal de Viçosa, 1986. 129p. Dissertação (Mestrado em Zootecnia) - Universidade Federal de Viçosa, 1986.

CHEN, P.; BASS, T.J.; DEKKERS, J.C.M. Selection for lean growth rate in a synthetic line of Yorkshire-Meishan pigs. 1. Selection pressure applied and direct response. In: Breeding/Physiology. Iowa: Iowa State University, 1994. 6p.

COLLAO SAENZ, E.A. Aplicação de modelos animais na estimação de parâmetros genéticos em características de carcaça de suínos. Lavras: Universidade Federal de Lavras, 1994. 57p. Dissertação (Mestrado em Zootecnia) Universidade Federal de Lavras, 1994.

COSTA, A.R.C.; LOPES, P.S., TORRES, R.A. et al. Estimação de parâmetros genéticos em características de desempenho de suínos das raças Large White, Landrace e Duroc. Revista Brasileira de Zootecnia, v.30, n.1, p.49-55, 2001.

CUTHBERTSON, A.; PEASE, A.R.H. The interrelationships of various measurements, visual assesments and dissection results of pigs of $200 \mathrm{lb}$ live weight. Animal Production, v.10, n.3, p.249-255, 1968.
CUTHBERTSON, A. PIDA Dissection techniques. In: SYMPOSIUM ON METHODS OF CARCASS EVAluAtion, 1968, Dublin. Proceedings...Dublin: European Association for Animal Production, 1968. 8p.

FOWLER, V.R.; BICHARD, M.; PEASE, A. Objectives in pig breeding. Animal Production, v.23, n.3, p.365-387, 1976.

HICKS, C.; TSUTOMU, F.; SCHINCKEL, A.P. Estimates of genetic parameters for daily gain and carcass traits for Japanese Large White Swine. Swine Day Report, 1998. 3p.

HOFER, A.; HAGGER, C.; KUNZI, N. Genetic evalution of onfarm tested pigs using an animal model. II. Prediction of breeding values with a multiple trait model. Livestock Production Science, v.30, p.83, 1992.

JOHNSON, Z.B.; CHEWNING, J.J.; NUGENT, R.A. Genetic Parameter for production traits and measures of residual feed intake in Large White. Journal of Animal Science, v.77, p.1679-1685, 1999.

LARZUL, C.; LEFAUCHEUR, L.; ECOLAN, P. et al. Phenotypic and genetic parameter for longissimus muscle fiber characteristics in relation to growth, carcass and meat quality traits in Large White pigs. Journal of Animal Science, v.75, p.3126-3137, 1997.

OLIVEIRA, A.I.G. Aspectos genéticos das características físicas das carcaças de suínos em cruzamentos dialélicos. Viçosa, MG: Universidade Federal de Viçosa, 1988. 97p. (Doutorado em Zootecnia) - Universidade Federal de Viçosa, 1988.

PATTERSON, H.D.; THOMPSON, R. Recovery of inter-block information when block sizes are unequal. Biometrika, v.58, n.3, p.545-554, 1971.

RAHNEFELD, G.W.; CLIPLEF, R.L.; GARNETT, I. et al. Mass selection for postweaning growth in swine. VI. Means, heritabilities, genetic and phenotypic correlations, and correlated response of carcass traits. Canadian Journal of Animal Science, v.63, n.1, p.49-58, 1983.

ROSO, V.M.; FRIES, L.A.; MARTINS, E.S. Parâmetros genéticos em características de desempenho e qualidade de carcaça em suínos da raça Duroc. Revista da Sociedade Brasileira de Zootecnia, v.24, n.2, p.310-316, 1995.

SONESSON, A.K.; GREEF, K.H.; MEUWISSEN, T.H.E. Genetic parameter and trends of meat quality, carcass composition and performance traits in two selected lines of Large White pigs. Livestock Production Science, v.57, p.23-32, 1998.

STERN, S.; JOHANSSON, K.; RYDHMER, L. et al. Performance testing of pigs for lean tissue growth rate in a selection experiment with low and high protein diets. 1. Experimental design and efficiency of selection. Acta Agriculturae Scandinavica, Seção A, Animal Science, v.43, n.3, p.1366-143, 1993. 